\title{
Development and Design of Ice and Snow Landscape System Based on Network and Information Technology
}

\author{
Wang Shun-hui and Lin Xue-wei* \\ Institute of Arts and Technology, \\ Harbin University of Science and Technology, China \\ *Corresponding Author Email:en.landscape@gmail.com
}

\begin{abstract}
Ice and Snow Art Landscape is unique urban landscape form in cold region. It is also artistic form reflecting the regional characteristics and cultural connotations as the symbol of the city. It has the important meaning for urban tourism industry and related industries. The construction of Urban Ice and Snow Art Landscape Information Database is an important channel promoting the development of Urban Ice and Snow Art. The research is based on modern network information technology, taking Urban Ice and Snow Art Landscape as research object. It puts forward on idea constructing Urban Ice and Snow Art Landscape Information Database, and design system architecture in the background using the technology of database, front design using DW, including Data Dictionary, E-R model, SQL, PHP and WEB publish. The development and Application of Urban Ice and Snow Art Landscape Information Database, is very necessary for the great-leap-forward development of Urban Ice and Snow Art Landscape.
\end{abstract}

Keywords: Ice and Snow Landscape, Urban Arts, Network, Information Technology

\section{Ice and Snow Landscape and Data Acquisition}

Ice and Snow Art is unique form which is formed in the formation of human production activities, in the need of human life in the north of frigid zones. In the process of meet the need of human aesthetic life, it produced brilliant civilization achievements. All of ice and snow and ice products containing skills and thinking activity can be called as Ice and Snow Art. Ice and Snow Art is the high level requirements in People's life, as the living standard gets higher, the demand for ice and snow art is more urgent.

\subsection{Urban Ice and Snow Art Landscape}

Ice and Snow Art Landscape is a unique landscape art form in North latitude 45 degrees Celsius. From the perspective of sociology, Ice and Snow Art Landscape is local art form satisfying the people spiritual needs and urban public environment beautification. It includes the following forms: ice carving, snow sculpture, ice and snow amusement park, ice and snow experience pavilion, and so on.

As the material elements, ice and snow art are the products of natural climate cold. For a long time, people use ice and snow as a kind of resources. With the development of Ice and Snow Festival in cold regional cities such as Harbin, Sapporo, Quebec, Helsinki and so on, Ice and Snow Art displays more rationality and more diverse cultural connotation. In the same time, with the development of Ice and Snow Art in the world, as the culture of the world, Ice and Snow Art has come into the stage of industrialization. This is also the beginning of marketization of Ice and Snow Art.[1]

Internet and information technology, in particular, the advent of the era of big data radically changed the lifestyle of modern people. The strategic significance of big data technology is not only in huge data information, but also in the professional analysis and 
processing of data with specific meanings. If the big data compared to an industry, the key to profit this industry is to improve data's processing ability, so as to realize the appreciation of data. The core value of the large data is storage and analysis for huge amounts of data. Compared with the other techniques, Big Data has the advantage of lowpriced, speed and optimization, therefore the sustainable development of all walks of life needs to rely on and large data and information technology. As Local art form and the main Attractions, it is the window of urban culture, needs to dock with big data era, raise the level of the internationalization of ice and snow art and the ability to serve the society.

\subsection{The Development of Urban Ice and Snow Art Landscape Information System}

The establishment and application management of the WEB site based on database, is very necessary for the creation and development of Ice and Snow Art landscape. It is very necessary for ice and snow art landscape creation.

The application of the database structure: 1.By MYSQL technology, we can create Urban Ice and Snow Art Database, create all kinds of related tables in the database, and according to user's need to get the corresponding information. 2. Using Variables, conditions and control structure of PHP, write database and provide HTML scripts to the user's browser. 3. DW visualization interface technology, set up and test server.

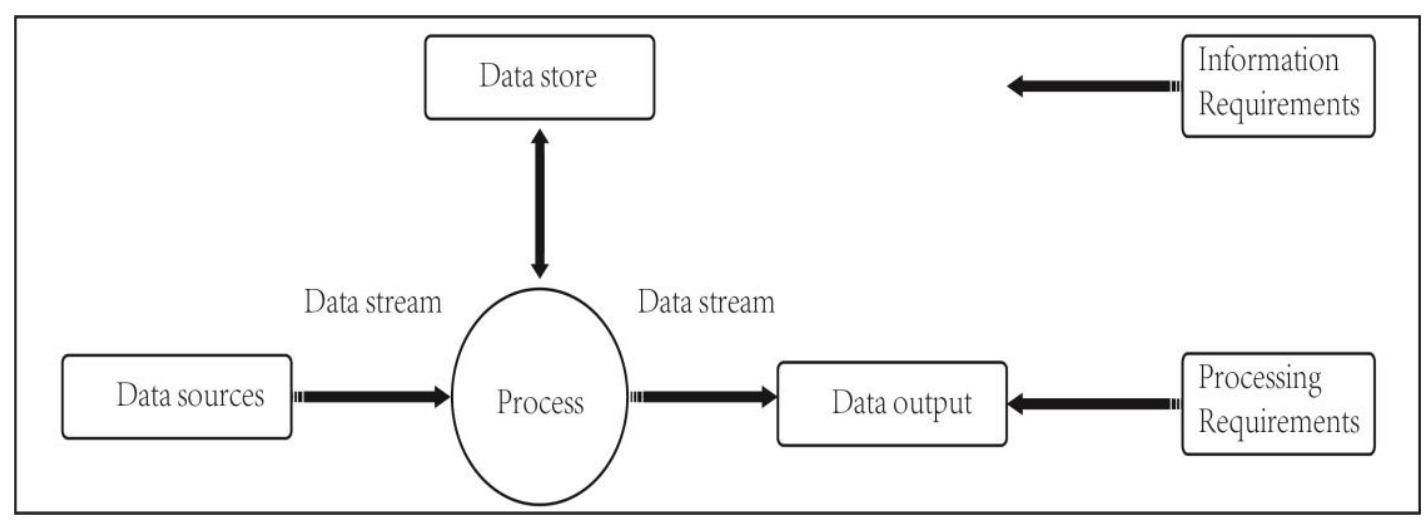

Figure 1. The Basic Structure of the Information Data Model

Data collection of Urban Ice and Snow Art Database has a certain particularity. On the basis of investigation and collection of Urban Ice and Snow Art Database, create Urban Ice and Snow Art Database and information inquiry management system. From the Angle of the practical application of the system, not only collected the general properties of ice and snow art landscape, but also add the artistic quality of Ice and Snow Art Landscape, Art building technology, Local tourism and cultural contribution analysis, contest analysis, Morphological image, and so on, fully embodies the practicality and the social value. This system used a flexible way of query, realize the information and standardization management. [2]It can provide a variety of reference for creators and the builders of Urban Ice and Snow Art Landscape, is very important to improve the awareness level and propaganda work of snow and ice landscape of the city. 


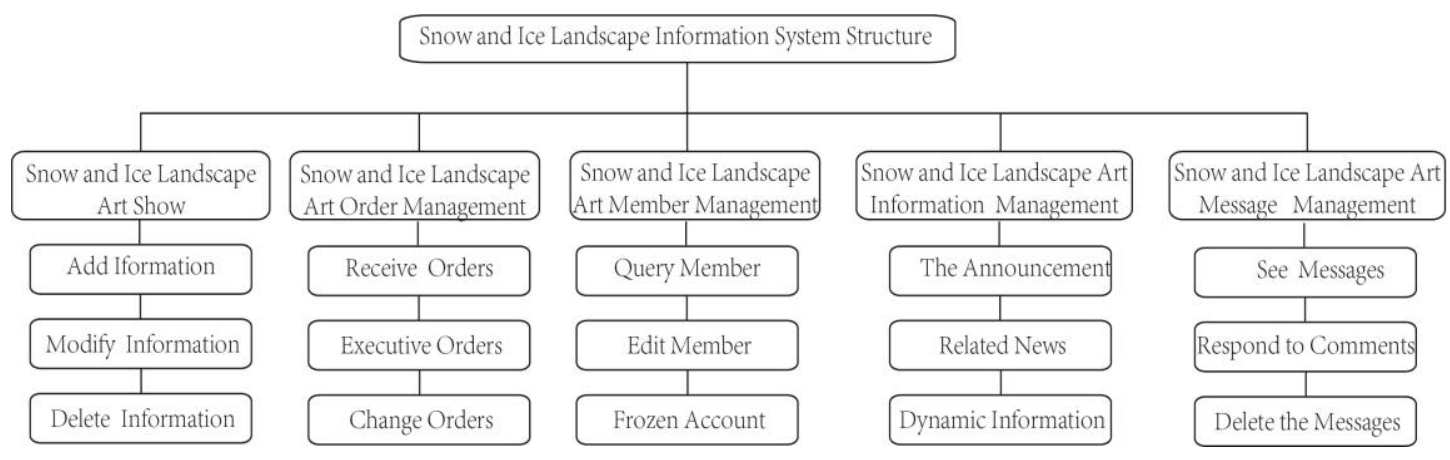

Figure 2. Structure of Snow and Ice Landscape Information System

\section{Construction and Design of Urban Ice and Snow Art Landscape Database}

Collect domestic high value and the typical city of ice and snow landscape resources to comprehensive information database, develop the management information database by modern information technology. The system has the main function including: data entry, query, statistics and analysis, picture browsing, system maintenance.[3] It ought to have following characteristics: comprehensive functions, user-friendly control, large amount of information data, fast data processing speed, flexible data output. application of the system offers comprehensive application database of Urban Ice and Snow Art Database for the landscape design industry users. The system architecture of the library should include the data source analysis, conception framework design, logical design, physical design and enforcement and maintenance.

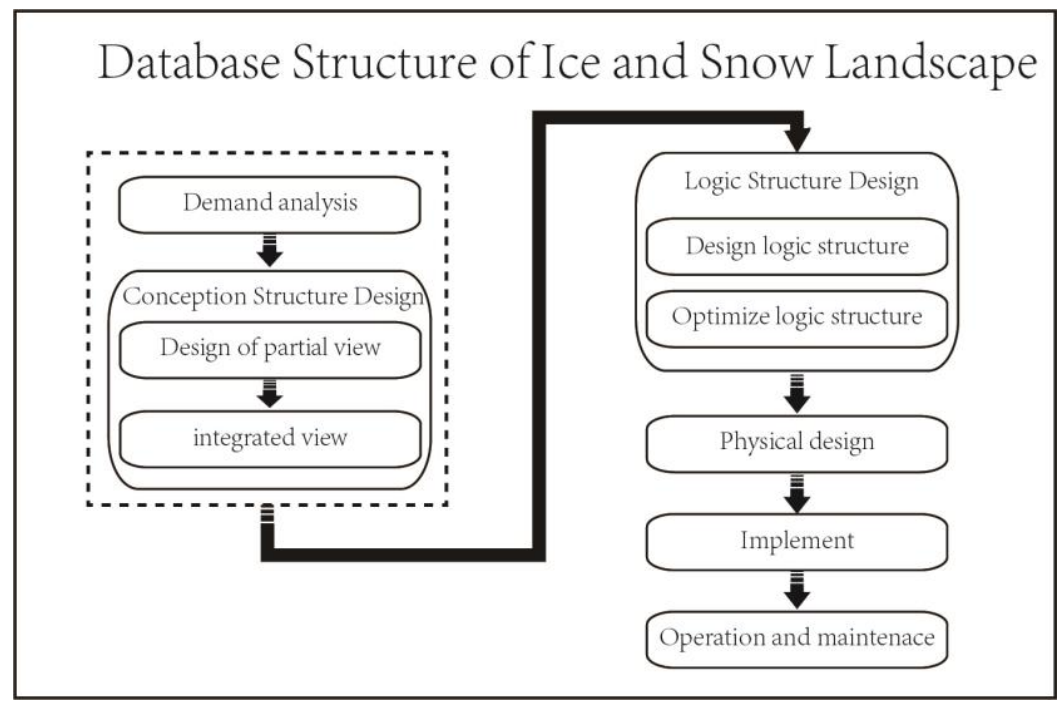

Figure 3. Database Structure of Ice and Snow Landscape

\subsection{Data Collection of Urban Ice and Snow Art Landscape Database}

The data collection of Urban Ice and Snow Art Landscape has following characters: large volume of data, multi category, complex structure. From the data form analysis, it includes quantitative numeric data, numeric text, graphics, image, AUDIO, video and Hypertext, etc. From Data acquisition, it includes on-line data acquisition, manual record, dynamic monitoring and so on. From the connotation of the data, it includes first-hand 
data, data processing after finishing, result data after professional statistical analysis processing.

Online acquisition is electronic data collection behavior about characteristic area' $\mathrm{s}$ Ice and Snow Art Landscape, including Landscape scene photos, aerial map, etc; manual record mainly refers to the scene photos, Audiotapes and Videotapes, transcript, Draw pictures; dynamic monitoring mainly refers to landscape growing principle, comprehensive landscape' s dynamic monitor and record. For example, plant scale morphology and color changes of the same project in different years, and coordination effect between the change and environmental factors.

\subsection{Conceptual Design of Urban Ice and Snow Art Landscape Database}

At the bottom of the Ice and Snow Art Landscape database, we set an Ice and Snow Art Landscape project composite foundation database for data input, data management, data analysis and result announcing. data acquisition, manual record, dynamic monitoring. By automatically collect data interface, monitoring data of automatic system will be input to composite foundation database, deal with the mobile data acquisition device files, Online input text by scanning way. Data Input should have following functions: validity check, data processing and transformation, gross error reject, complementary error function, in order to ensure the reliability of the incoming information. According to the specific needs, we can select the server-side trigger technology or application programming processing.

The application of data management subsystem is based on database, should offer iquery function of item information, landscape degree, actual photos, resident participation, field experience, safe and reliable additive, delete and modify functions, production specification requirements, all kinds of user's customization, including professional statistical statements, professional drawing graphics, Audio and video media information, and so on. The above not only need rapid and reliable data interface between the application and the database, but also need concise and complete data structure.

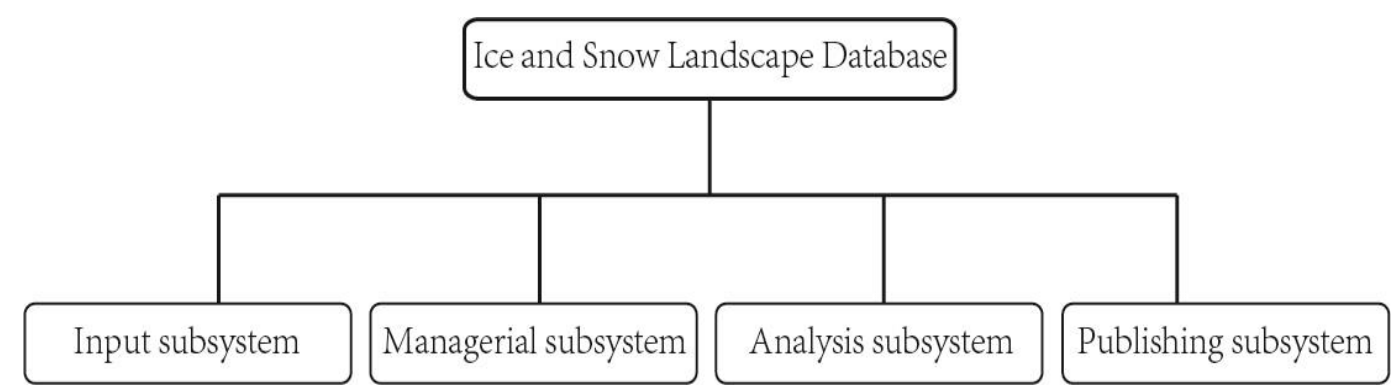

Figure 4. Concept Design of Ice and Snow Landscape Database

About data analysis subsystem, database should offer model, knowledge and correlation parameter, such as Ice and Snow Art professional model analysis, comprehensive analysis online, offline analysis, expert consultation. These data should be open to users, in order to query, maintain and expand. The database also need intermediate information storage, in order to exchange and sharing data. Result announcing subsystem should have following functions: monitoring results, analysis result, expert suggestion, will be released to the world through WEB. This poses a challenge to the interface between database and web server.

To sum up in conclusion, Ice and Snow Art Landscape database based on underlying database, should includes following content: 1 . Project collection database, which stores data about urban ice and snow projects. 2 . Raw data base, storing raw data automatically collected or manual record data. 3. Data reorganization, storing the project data reorganized. 4. Achievement database, storing comments and suggestions after attribute 
decomposition and statistic analyses for city managers, policy makers, Ice and Snow Art Landscape developers, Ice and Snow Art framers and customers.[4]

The composition and structure is logic design concept. On the physical implementation, all the data should be store in one or a few actual database, for the design of data relations and data correlation operation. In view of the system has the function of each part needs of users, view technologies can be used to show independent complete professional database.

\subsection{The Logic Design of Urban Ice and Snow Art Landscape Database}

The logic design of Ice and Snow Art Landscape Database is the core of the relational database system optimization design. Logic design is mainly for database tables and the relationship between the table and table design. Good logic design is the foundation of database and application performance optimization. Logical database design stage should finish the task as follows: found data dictionary, construct E-R model, definite object naming conventions, definite object naming conventions.

Data dictionary of Ice and Snow Art Landscape Database as table metadata table, according to user' $\mathrm{s}$ needs, The database table structure will be designed according to the metadata table, The table contents will be changed into the SQL statement, in order to database. With some engineering database integrate data table as an example, data dictionary and SQL statements are presented.

Table 1. Column Metadata

\begin{tabular}{|c|c|c|c|c|c|c|}
\hline Field name & Length of type & Null value & Meaning & Main code & Outer code & Instructions \\
\hline Table number & CHAR & NOTNULL & & $\checkmark$ & $\sqrt{ }$ & \\
\hline Table type & CHAR & NOTNULL & & $\sqrt{ }$ & & \\
\hline English table name & VARCHAR & NOTNULL & & & & \\
\hline Chinese table name & VARCHAR & NOTNULL & & & & \\
\hline Column type & VARCHAR & NOTNULL & & & & \\
\hline Column width & CHAR & NOTNULL & & & & \\
\hline Unit & CHAR & NULL & & & & \\
\hline Primary key & CHAR & NOTNULL & & & & \\
\hline Null value & CHAR & NOTNULL & & & & \\
\hline Value range & VARCHAR & NULL & & & & \\
\hline Note & VARCHAR & NULL & & & & \\
\hline
\end{tabular}

E-R Model is important database design tools, Its constitute the basic elements is entity type, attribute and contact, then change into relation model. The E-R Model of Urban Ice and Snow Art Landscape Database is shown as following figure 5. 


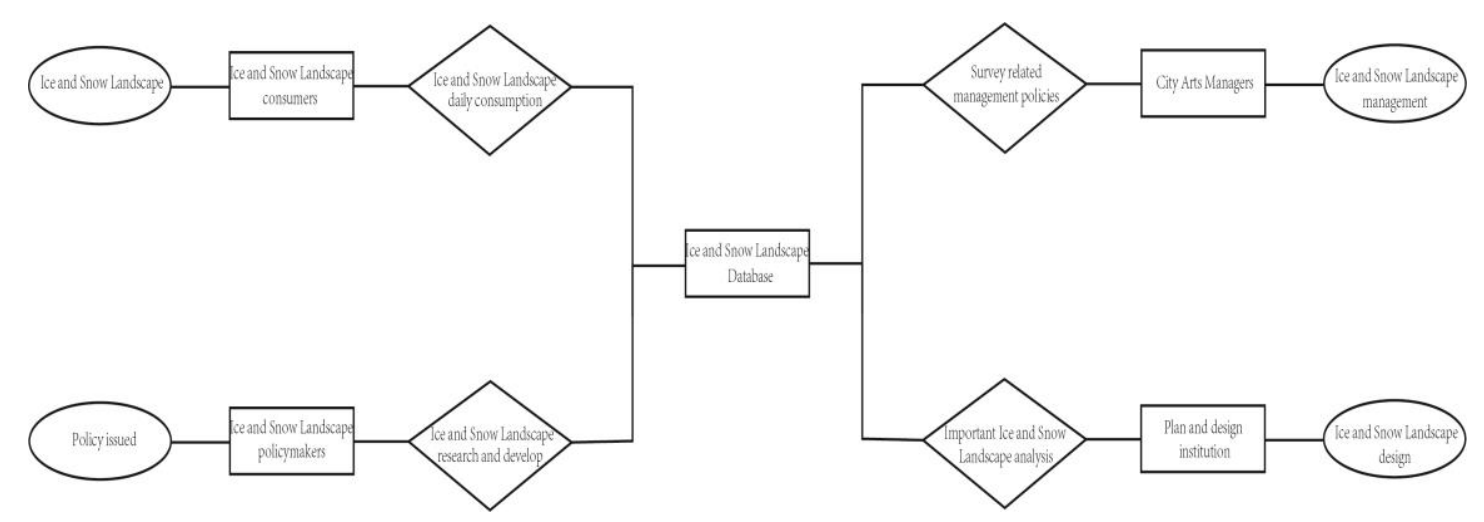

\section{Figure 5. The E-R Model of Ice and Snow Landscape Database System}

\subsection{The Physical Design of Ice and Snow Landscape Database}

Based on logic design, the physical design of Ice and Snow Art Landscape Database designs storage structure and access method, in order to ensure that the database things run efficiently. Physical design on the one hand, rely on computer systems, on the other hand, is related to the database management system (DBMS), Including the field type, Length, accuracy, DBMS data, page size, response time, stored memory allocation and the index selection, etc.

The hardware and software of Physical design, of course including the selection of DBMS above, should fully consider capacity on the efficiency of application system security and so on to meet demand. [5]DBMS is generally chosen in Oracle, DB2, SQL database and other well-known products according to need. The process of physical database building will classify the data, especially distinguish multimedia data such as dynamic data, static data and large binary objects, monitoring updating of data and data update slowly, in order to achieve quick query to modify data such as execution efficiency of the operation.

\subsection{Develop and Maintain Procedures of Urban Ice and Snow Art Landscape Database}

In view of the importance of Urban Ice and Snow Art landscape, it is very important to the reliability of the database construction, especially data collection and query and statistical analysis of Ice and Snow Landscape engineering in the typical representative area, and Ice and Snow Landscape Art projects of in the old streets of the old city. The stability of the system is particularly important, we can guarantee from the following aspects: firstly, choose a mature and stable hardware system; secondly, use data backup and recovery system with high safety, so that the inevitable hardware failure occurs, realize the data recovery, to avoid greater losses. [6]

\section{Development and Application of the Database Network Platform}

\subsection{WEB Data Release and Query}

The ultimate goal of snow and ice landscape information database construction is multi-user WEB use. The urgency and high quality of Urban Ice and Snow Art Landscape need city planners, Snow and Ice Landscape developer and landscape consumers to master data in a timely manner. We choose Dreamweaver for site connection release maintenance and daily management work. 
The value of WEB application is based on database user query and database updates. This determines the WEB design must choose a dynamic WEB technology. The dynamic web page does not mean the dynamic effect, it is the data dynamic exchange between user and the system. [7]The site made up of dynamic web usually based on database technology. Using dynamic language realizes database interactions, so as to achieve the purpose of the data update, at the same time also greatly reduces the site maintenance work. Dynamic web pages needs the support of the server. The files end with HTML can be opened by double-click. But dynamic web pages are different, we need a server for support, at the same time, the server must also support the dynamic languages to browse.

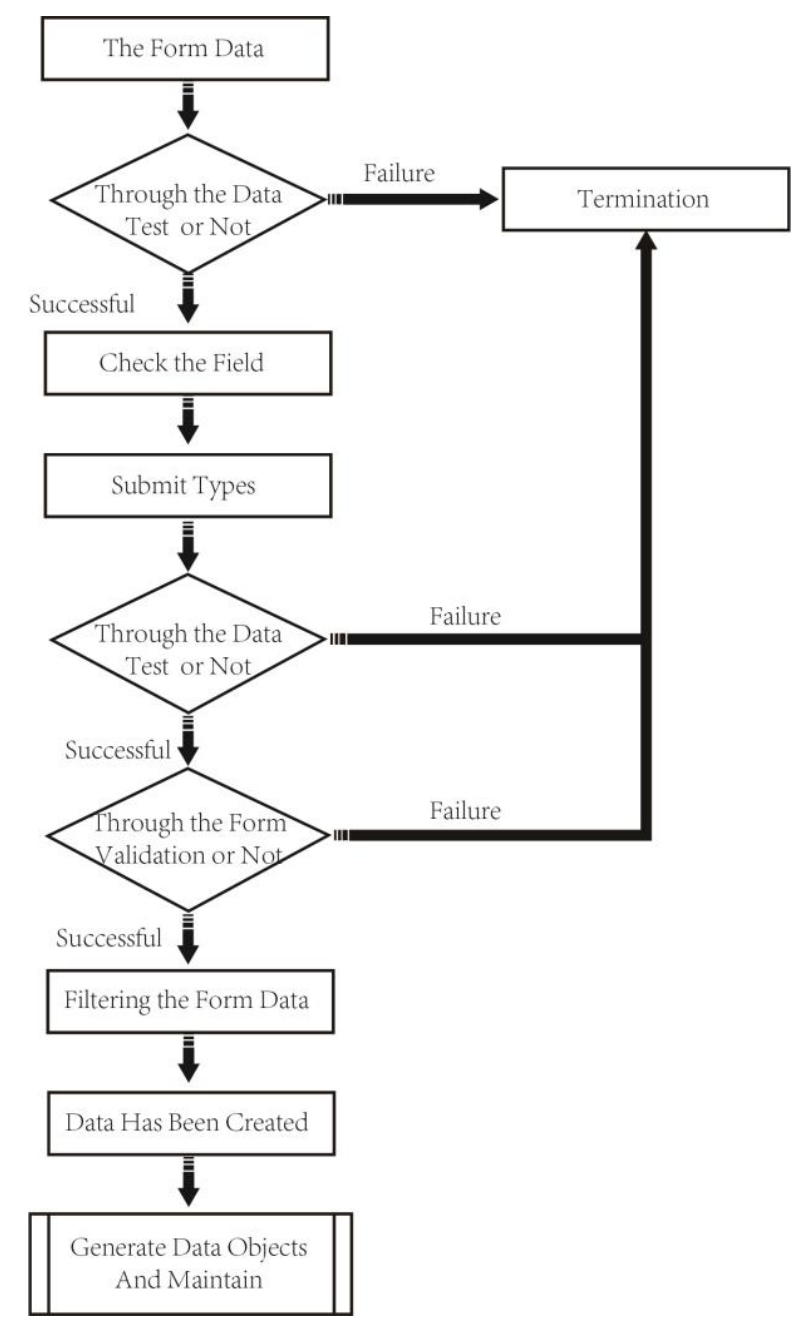

Figure 6. Model of Ice and Snow Landscape System Data Flow

\subsection{The Collocation of the Development Environment}

The specific application of Urban Ice and Snow Art Landscape Database needs to match the development environment. The server can use Windows own IIS server, also can use the more popular Apache server, it is determined by the dynamic language technology. ASP corresponds to IIS, and PHP chooses Apache. The choice of database needs to choose according to dynamic language. If choose ASP, we need ACCESS database with the match; if choose PHP, we need MYSQL database with the match. After years of development practices, we recommend the combination of PHP+MYSQL. Then we need combine dynamic language and database server. Development environment collocation is indispensable to realize dynamic Web page. [8] In the release phase, in the 
steps of test release management, DreamWeaver will be used with a combination of PHP+MYSQL, and it has good effect.

\section{Conclusion}

Big data era are raised informatization requirement for various industries. The construction of Urban Ice and Snow Landscape Database, is the important foundation and demand of the time. The collection and analysis of landscape data, will become the important basis of urban planning, is the comprehensive implementation of modern garden city and ecological city construction. In the city of Ice and Snow Art Landscape Database construction, this paper proposed the combined way of PHP + MYSQL and DreamWeaver. We hope to provide some useful reference to achieve the informatization of Urban Ice and Snow Art Landscape construction.

\section{Acknowledgements}

This research supported by Philosophy and Social Sciences Planning project of Heilongjiang Province (Project No.14E062). The author is director of Institute of Arts and Technology, Harbin University of Science and Technology, research in the field cross of arts, Technology and social sciences.

\section{References}

[1] C. Georg, "The Metropolis and Mental life:center and symbol of our times", New York University Press, (1995).

[2] L. S. Pan, "Demystifying Case Research:A Strucured-Pragmatic-Situational(SPS) Apprach to Conductiong Case Studies", Information and Organization, vol. 3, no. 21, (2011).

[3] N. Levina and E. Vaast, "The Emergence of Boundary Spanning Competence in Practice", Implications for Implementation and Use of Information Systems, vol. 2, no. 29, (2004).

[4] H. N. Duan and D. Y. Chen, "Workflow Management System Based on SOA", Journal of Harbin University of Science and Technology, vol.14, (2009), pp.65-71.

[5] S. Gershman, "A Tutorial on Bayesian Nonparametric Models", Journal of Mathematical Psychology, vol. 1 , no. 56, (2012)

[6] T. Hastie and R. Tibshirani, "The Eements of Statistical Learning”, Springer, (2009).

[7] S. H. Wang and J. Zou, "Construction and Application of Information Base of Urban Residential Landscape Based on Big Data", International Journal of Smart Home, vol. 9, no. 5, (2015).

[8] J. G. Anthony, "The Fourth Paradigm: Data-Intensive Scientific Discovery", Microsoft Research, (2009). 